\title{
Experimental and Clinical Evaluation of the Harmonic Scalpel in Thoracic Surgery
}

\author{
AKIHIRO HAYASHI, SHINZO TAKAMORI, TOSHIHIRO MATSUO, KOHSUKE TAYAMA, \\ MASAHIRO MITSUOKA AND KAZUO SHIROUZU
}

Department of Surgery, Kurume University School of Medicine, Kurume 830-0011, Japan

\begin{abstract}
Summary: The Harmonic Scalpel is an ultrasonic instrument for cutting and coagulating tissue. We are reporting our evaluation of the Harmonic Scalpel safety and efficacy in both experimental and clinical thoracic surgery. First, we confirmed the safety in thoracic surgery by following two preliminary studies using the Harmonic Scalpel. 1: Pulmonary parenchyma was incised using "Coagulating Shears" to evaluate hemostasis and air leakage. 2: Pulmonary hilar vessels were contacted directly with "Dissecting Hook" blade at optimum cutting power mode to evaluate potential vascular wall injury by the Harmonic Scalpel. Subsequently, the Harmonic Scalpel was used for a partial lung resection due to metastatic lung cancer. Particular application was for a chest wall incision, interlobar separation of the lung, and dissection of a pulmonary artery, in lung cancer operations. We concluded that cutting and hemostasis of pulmonary parenchyma could be achieved with minimal tissue damage using the Harmonic Scalpel. Compared to electric coagulation, the Harmonic Scalpel minimizes tissue charring and dissection, and eliminates thermal injury in thoracic surgery.
\end{abstract}

Key words Harmonic Scalpel, thoracic surgery, lung cancer, thoracoscopic surgery, VATS

\section{INTRODUCTION}

The "Harmonic Scalpel" is an ultrasonic instrument for cutting and coagulating tissue, originally developed for use in abdominal surgery and especially for use in laparoscopic surgery [1-3]. Here we report our evaluation of its safety and efficacy in experimental and clinical thoracic surgery.

\section{ANIMAL EXPERIMENT}

\section{Materials and Methods}

Cutting and coagulating procedures were performed with an ultrasonically activated scalpel (Harmonic Scalpel, Ultracision, Smithfield, RI, USA) in the following animal experiments and clinical operations.

A pig weighing $35 \mathrm{~kg}$ was used for the experi- mental evaluation. Thoracotomy was carried out with endotracheal intubation and with controlled ventilation under intravenous anesthesia. Two investigative studies were done as follows.

First, cutting of pulmonary parenchyma was performed using "Coagulating Shears" of the Harmonic Scalpel by means of "grasping technique," (Fig. 1) for the purpose of evaluating hemostatic condition and air leakage.

Second, direct contact to pulmonary hilar vessels with "Dissecting Hook" blade of the Harmonic Scalpel at optimum power setting was performed for the purpose of evaluating vascular (pulmonary artery, pulmonary vein, superior vena cava and aortic arch) wall injury.

This animal experiment was carried out in the laboratory of ETHICON ENDO-SURGERY in Japan (Johnson \& Johnson Medical Japan Corp., Sukagawacity, Fukushima, Japan). The animal received 

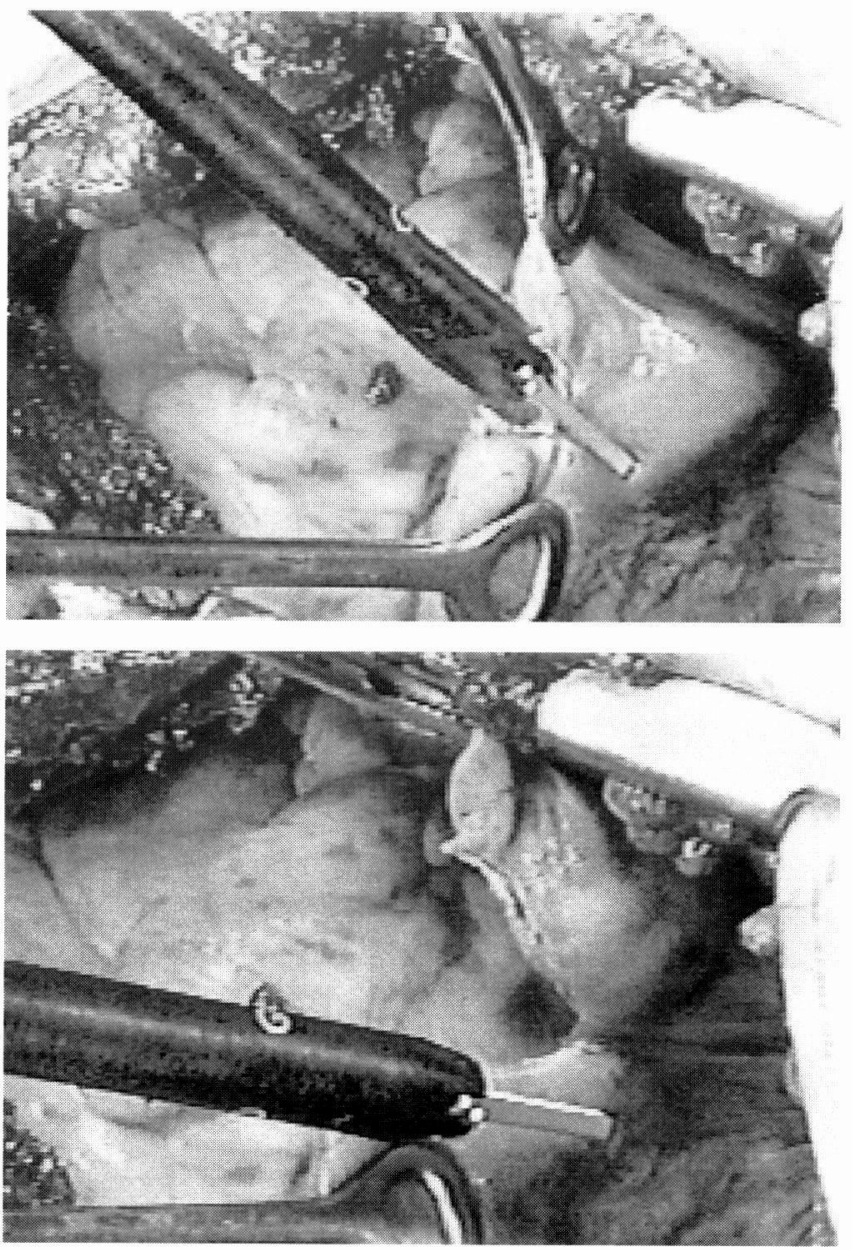

Fig. 1. Cutting porcine pulmonary parenchyma by means of "Grasping technique" with Coagulating Shear (CS) Blades. humane care in compliance with the "Principles of Laboratory Animal Care" formulated by the National Society for Medical Research. Care was also given in compliance with "Guide for the Care and Use of Laboratory Animals" prepared by the Institute of Laboratory Animal Resources and published by the National Institute of Health.

\section{Results}

The mean cutting time of one "Coagulating Shear" (15 mm length) of lung parenchyma with grasping

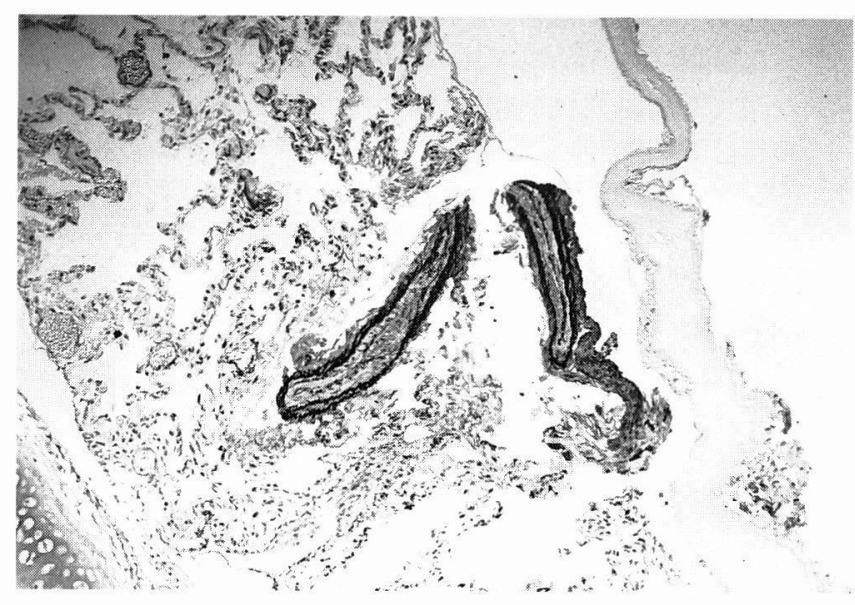

Fig. 2. Microscopic appearance of pulmonary parenchyma cut with Shear mode of CS Blades (power level 5). (Elastica van Gieson stain $\times 400$ ) Thermal damage is minimal and the vessel is sealed by adhesive coagulum.

TABLE 1

Cutting of the pulmonary parenchyma using "Coagulating Shear" (CS) Blade in porcine model

\begin{tabular}{lccccc}
\hline Mode & $\begin{array}{c}\text { Power } \\
\text { level }\end{array}$ & $\begin{array}{c}\text { Duration of } \\
\text { cutting*(sec.) }\end{array}$ & $\begin{array}{c}\text { Thermal damage } \\
\text { of lung** }\end{array}$ & $\begin{array}{c}\text { Hemostatic } \\
\text { effect }\end{array}$ & Air leakage \\
\hline Shear & level 3 & 6.8 & + & fair & + \\
& level 5 & 2.0 & - & fair & + \\
Blunt & level 3 & 9.0 & ++ & fair & + \\
& level 5 & 3.0 & fair & + \\
Flat & level 3 & 18.3 & + & fair & + \\
& level 5 & 7.5 & + & fair & + \\
\hline
\end{tabular}

* mean cutting time with grasping technique-one CS blade length $(15 \mathrm{~mm})$

** Thermal damage was graded microscopically from - (none) to + (severe) 
technique (Fig. 1) by shear mode in power level 3 and in power level 5 was $6.8 \mathrm{sec}$ and $2.0 \mathrm{sec}$. That of by blunt mode, in power level 3 and in power level 5 was $9.0 \mathrm{sec}$ and $3.0 \mathrm{sec}$. By flat mode, in power level
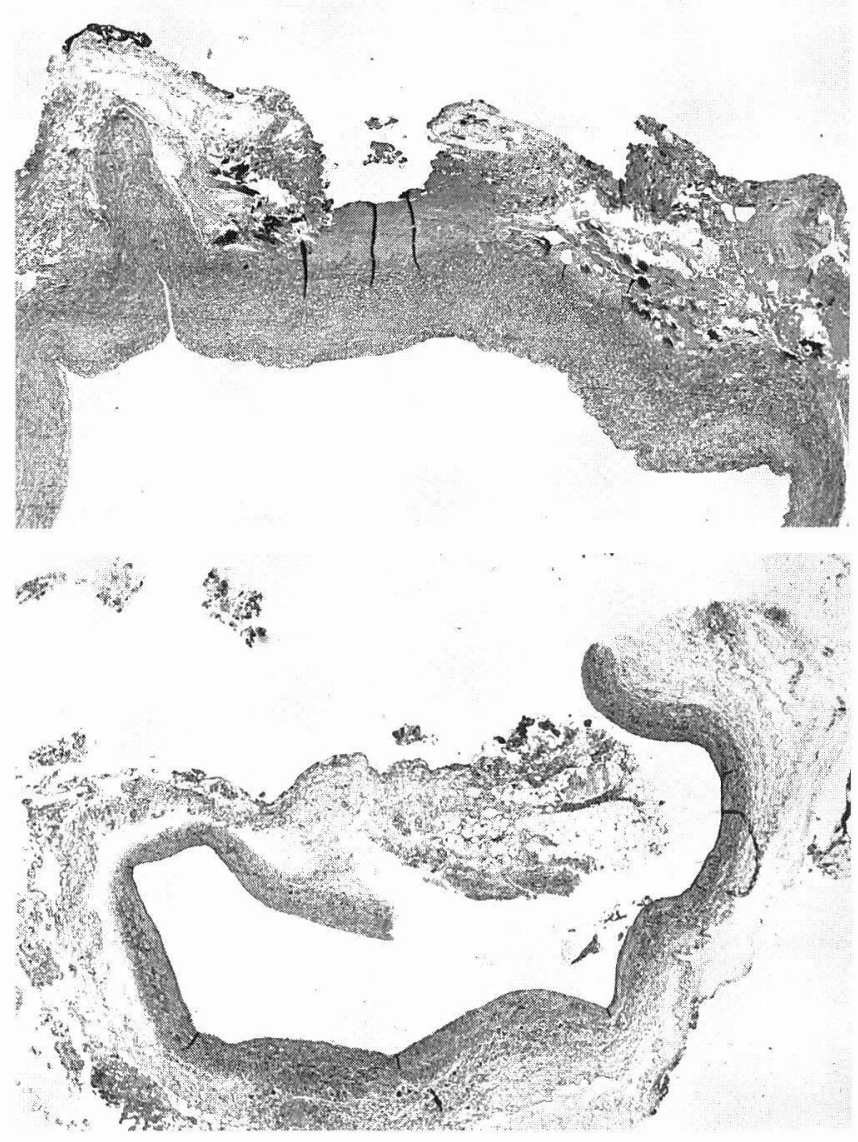

Fig. 3. Microscopic appearance of vascular wall contacted with "Dissecting Hook" Blade in porcine model. (Hematoxylin and eosin stain $\times 400$ ) Thermal damage of vascular wall is minimal. Main trunk of pulmonary artery was not perforated by contact with highest power cutting mode in $30 \mathrm{sec}$ duration (top). Superior pulmonary vein was perforated by contact with highest power cutting mode in $20 \mathrm{sec}$ duration (bottom).

TABLE 2.

Contacting directly to intra thoracic large vessels with "Dissecting Hook" Blade in porcine model

\begin{tabular}{lcc}
\hline & $\begin{array}{c}\text { duration of } \\
\text { contact* (sec.) }\end{array}$ & perforation \\
\hline Superior Vena Cava & 18 & + \\
Superior Pulmonary Vein & 20 & + \\
Main Pulmonary Artery & 30 & - \\
Thoracic Aorta & 30 & - \\
\hline
\end{tabular}

* Duration of contact to the vessels with the highest power cutting mode
3 and in power level 5 was $18.3 \mathrm{sec}$ and $7.5 \mathrm{sec}$, respectively (Table 1). The microscopic grade of thermal damage to the incised edge of the lung was associated with increasing of cutting duration. The hemostatic effect was almost gratifying in each mode and at each power level setting. However, air leakage from cut surface of pulmonary parenchyma was observed in every condition (Fig. 2).

The results of the second studies in regard to contacting directly to great vessels with "Dissecting Hook" blade were illustrated in Table 2. Both main pulmonary artery and aortic arch were not perforated by contact with highest power cutting mode in $30 \mathrm{sec}$ duration. However, superior pulmonary vein and superior vena cava ruptured in $20 \mathrm{sec}$ and $18 \mathrm{sec}$ respectively. Thermal damage of the vessel wall was not notable in every case microscopically (Fig. 3).

\section{CLINICAL APPLICATION}

The "Coagulating Shear" of the Harmonic Scalpel was used for a partial resection of a lung. "Dissecting Hook" blade was used for a chest wall incision, for interlobar separation of the lung, and for dissection of a pulmonary artery, during lung cancer operations and this balde was also used for excision of anterior mediastinal tumor involving superior vena cava and left subclavian artery (Fig. 4).

The Harmonic Scalpel was also used in videoassisted thoracic surgery with $5 \mathrm{~mm}$ "LaparoSonic Blade System" for resection of a chest wall tumor (neurilemmoma) arising from intercostal nerve. Resection of an anterior mediastinal tumor (teratoma) adjacent to superior vena cava, and resection of mediastinal tumor (neurilemmoma) adjacent to aortic arch were completed along with pleural adheolysis using "Coagulating Shears" (Fig. 5).

\section{DISCUSSION}

The Harmonic Scalpel is an ultrasonic surgical instrument for cutting and coagulating tissue, originally developed for use in abdominal surgery and especially for use in laparoscopic surgery [1-3]. The hand piece houses the ultrasonic transducer which creates mechanical vibration. This results in vibration of the transducer at its natural harmonic frequency of $55,500 \mathrm{~Hz}$.

The Harmonic Scalpel denatures protein by the transferring mechanical energy to the tissue and thus by generating heat from internal cellular friction which results from the high frequency of the tissue 

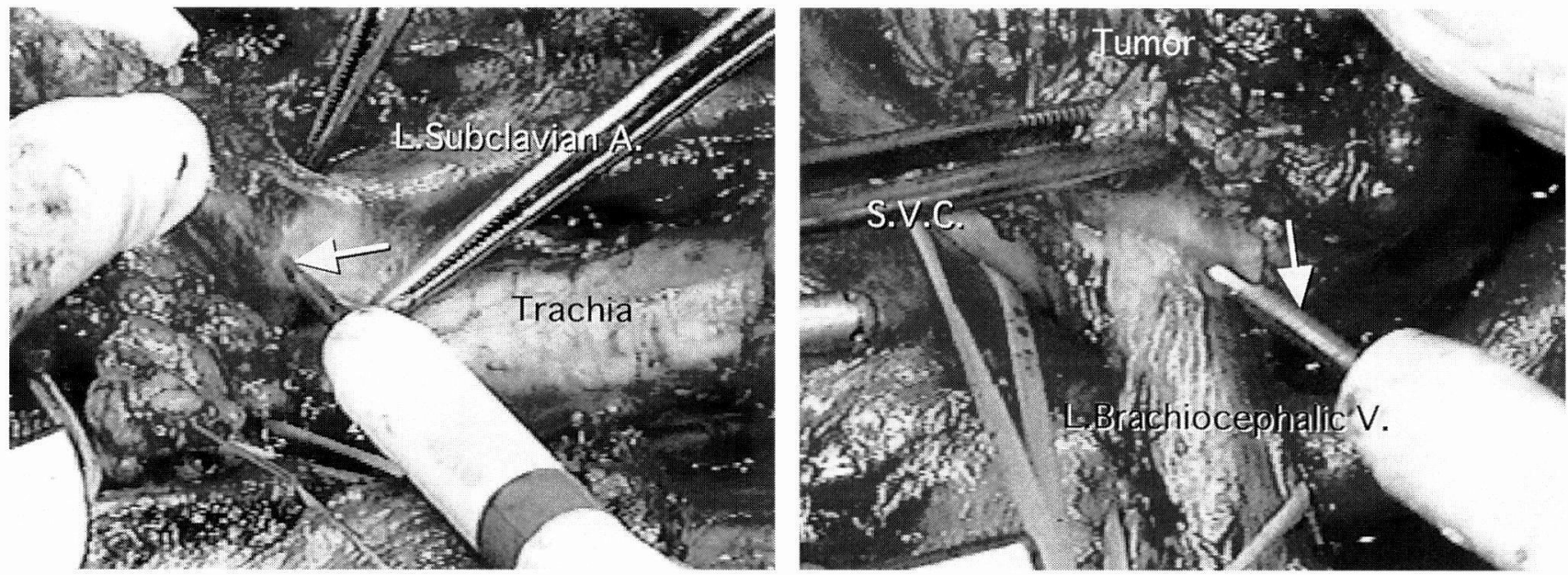

Fig. 4. Peri-vascular dissection during excision of anterior mediastinal tumor involving S.V.C. and left subclavian artery using "Dissenting Hook" Blade of the Harmonic Scalpel (white arrows). The vascular wall was not injured.
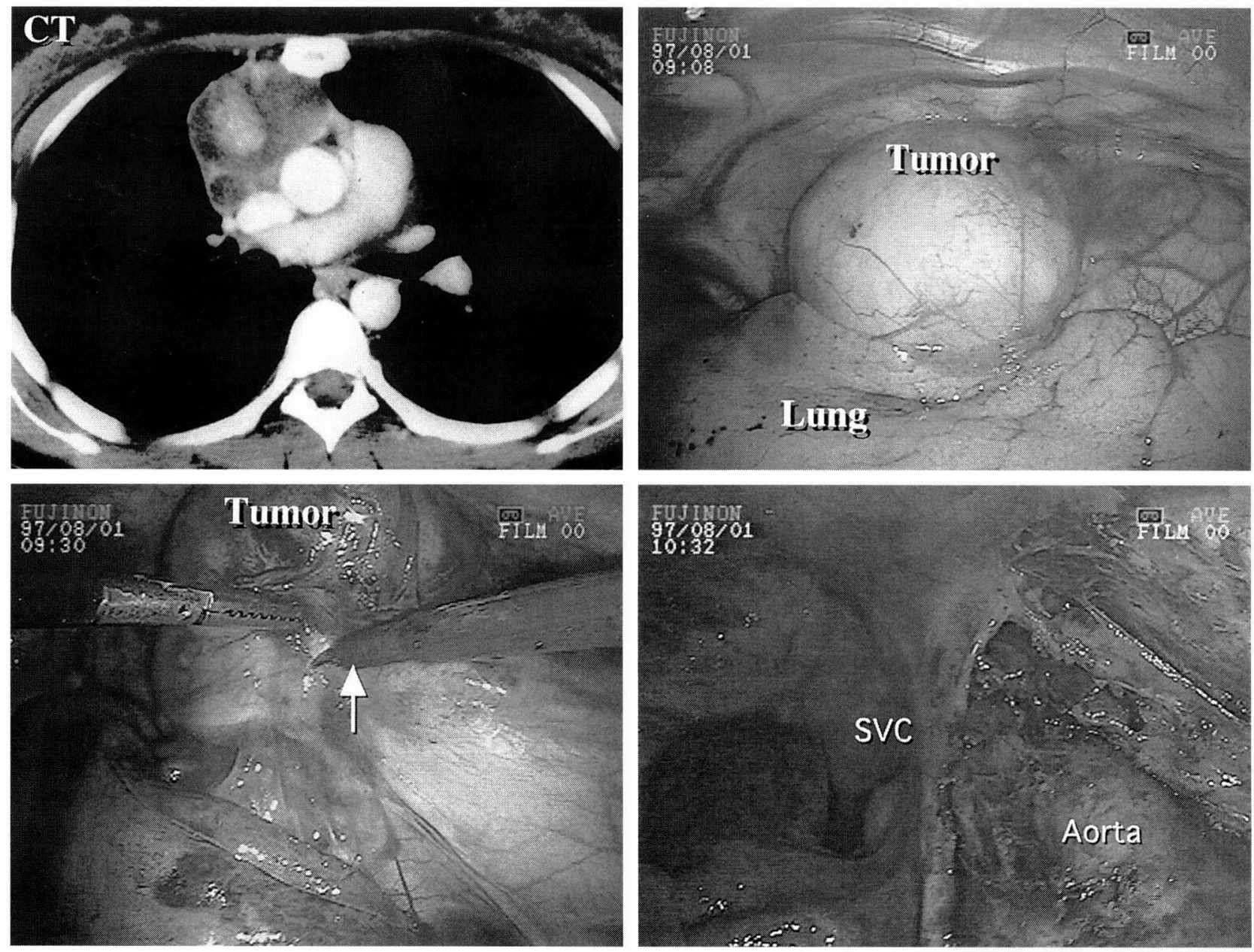

Fig. 5. Thoracoscopic excision of anterior mediastinal tumor (mature teratoma) adhering to pericardium and S.V.C. using "Dissecting Hook" Blade of the Harmonic Scalpel (white arrow in the left bottom). The right bottom shows post excision view. 
movement.

Each type of working blade travels from 50 to 100 microns per stroke, and rapid acceleration of tissue moving with the blade breaks the hydrogen bonds that give protein structure. This results in a protein coagulum that seals the cut vessels.

Incision is performed by the blade mechanically cutting the tissue by vibrating action. Coagulation is performed by heat generating friction which denatures protein and produces adhesive coagulum which seals vessels. Cavities are formed in the tissue by evaporating water located in the intra- and intercellular spaces, this action of cavitation facilitates cell wall destruction and incision.

Our result of this animal experiment has demonstrated that the Harmonic Scalpel is a safe device in thoracic surgery and we find that the advantages of the Harmonic Scalpel in thoracic surgery are as follows: (1) The Harmonic Scalpel causes less damage to the pleural surface and less air leakage when performing pleural adheolysis than does electrocautery of the Harmonic Scalpel makes an incision only the direction of detachment (blade tip) without thermal damage to the neighboring tissues. (2) The Harmonic Scalpel has the following advantages over the electrocautery in dissection of pulmonary interlobar as regard to better hemostasis, less air leakage, safer dissection around the pulmonary artery and facility in confirmation of interlobar fissure by cavitation effect. (3) The Harmonic Scalpel is superior to electrocautery regarding better hemostasis in case of pulmonary partial resection or segmentectomy, however we found no advantage in prevention of air leakage. (4) The Harmonic Scalpel does not generate smoke or tissue charring so an incision can be easily performed as the surgical margin has been recognized, in combined resection for the lung cancer case which has chest wall invasion. Especially in superior sulcus lung cancer case, apical chest wall resection can be performed with the Harmonic Scalpel safer than with the electrocautery, because of less thermal damage to the neighboring tissues and less risk of perforation of large vessels (such as the subclavian artery, vein and brachial nerve plexus) [4].

In addition, the Harmonic Scalpel is useful in thoracoscopic surgery as follows: (1) The operative view in the narrow thoracic space is not obstructed because the Harmonic Scalpel generates little smoke while cutting the tissue. (2) Incision and dissection of pulmonary interlobar can be performed safely with the Harmonic Scalpel because of little damage to large vessels (e.g., interlobar pulmonary arteries). (3) Excision of chest wall tumor including parietal pleura and intercostal muscles can be performed using the Harmonic Scalpel without injuring the intercostal arteries and nerves.

We conclude that cutting and hemostasis of pulmonary parenchyma could be achieved with minimal tissue damage using the Harmonic Scalpel. In comparison with electric coagulation, the Harmonic Scalpel minimizes tissue charring dessication and eliminates thermal injury. We considered that the Harmonic Scalpel will become a more effective surgical device not only in thoracoscopic surgery but also in open thoracic surgery in the near future.

\section{REFERENCES}

1. Amaral JF. The experimental development of an ultrasonically activated scalpel for laparoscopic use. Surg Laparosc Endosc 1994; 4:92-99.

2. Amaral JF. Ultrasonic dissection. Endoscopic Surgery \& Allied Technology 1994; 2:181-185.

3. Swanstrom LL, and Pennings JL. Laparoscopic control of short gastric vessels. J Am College Surgeons 1995; 181:347-351.

4. Hambley R, Hebda P, and Abell E. Wound healing of skin incisions produced by ultrasonically vibrating knife, scalpel, electrosurgery, and carbon dioxide laser. J Dermatol Surg Oncol 1988; 14:11. 